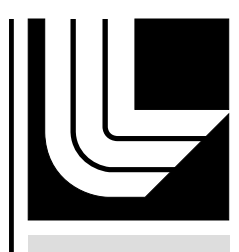

LAWRENCE LIVERM ORE N A TIO NAL LABORATORY

\title{
HPC4Energy Wrapup Report - LDRD 12-ERD-074
}

E. Dube

January 14, 2014 
This document was prepared as an account of work sponsored by an agency of the United States government. Neither the United States government nor Lawrence Livermore National Security, LLC, nor any of their employees makes any warranty, expressed or implied, or assumes any legal liability or responsibility for the accuracy, completeness, or usefulness of any information, apparatus, product, or process disclosed, or represents that its use would not infringe privately owned rights. Reference herein to any specific commercial product, process, or service by trade name, trademark, manufacturer, or otherwise does not necessarily constitute or imply its endorsement, recommendation, or favoring by the United States government or Lawrence Livermore National Security, LLC. The views and opinions of authors expressed herein do not necessarily state or reflect those of the United States government or Lawrence Livermore National Security, LLC, and shall not be used for advertising or product endorsement purposes.

This work performed under the auspices of the U.S. Department of Energy by Lawrence Livermore National Laboratory under Contract DE-AC52-07NA27344. 


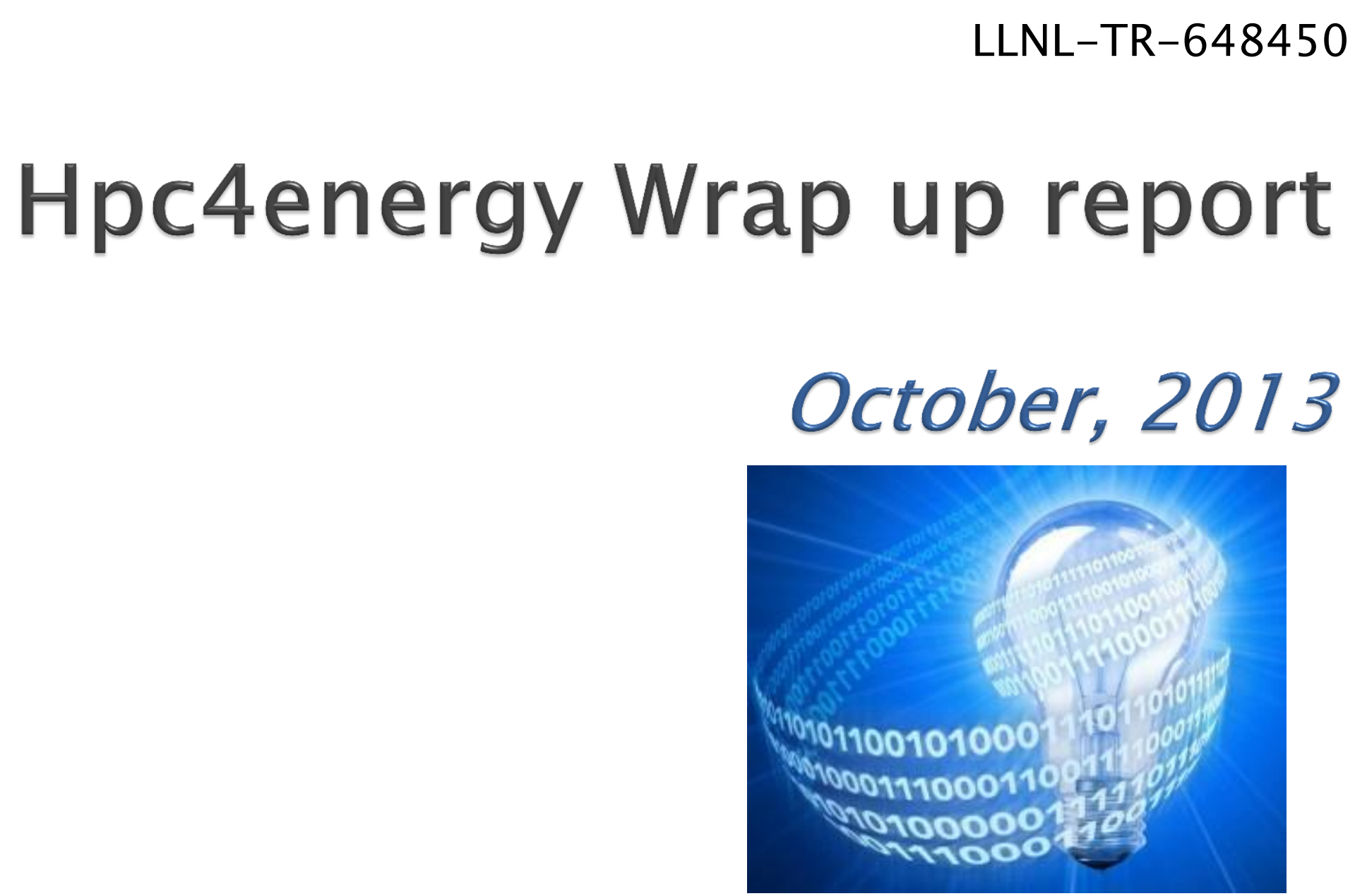

Lawrence Livermore National Laboratory

This work was performed under the auspices of

the U.S. Department of Energy by Lawrence

Livermore National Laboratory under Contract DE-

AC52-07NA2 7344. 
Improving Simulations of Advanced Internal Combustion Engines hpc4energy incubator wrapup October 2, 2013

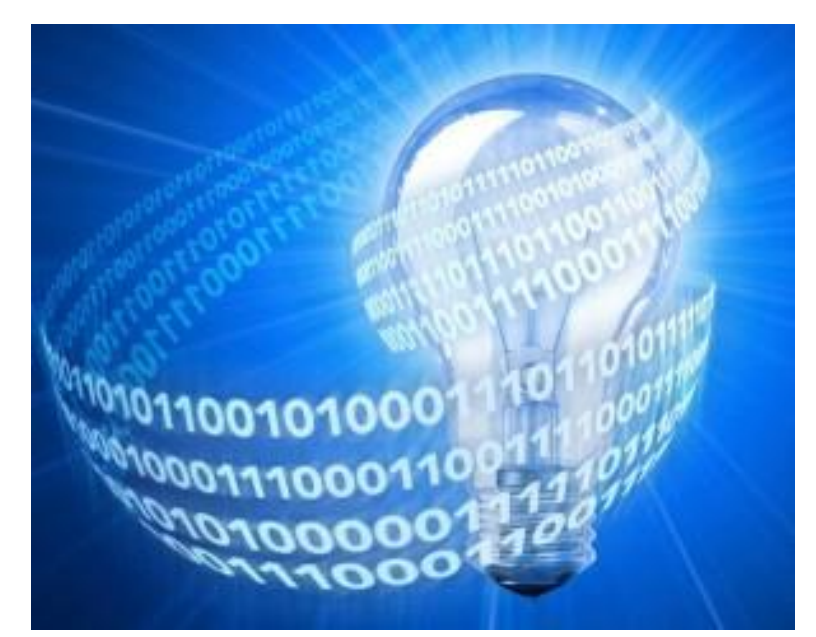

Robert Bosch LLC

in collaboration with

Lawrence Livermore National Laboratory 


\section{Robert Bosch}

\begin{tabular}{l|l|l} 
Est. & $\begin{array}{l}\text { Employee } \\
\mathbf{s}^{1}\end{array}$ & $\begin{array}{l}\text { Sales } \\
(\text { USD } / y r)^{1}\end{array}$ \\
\hline
\end{tabular}

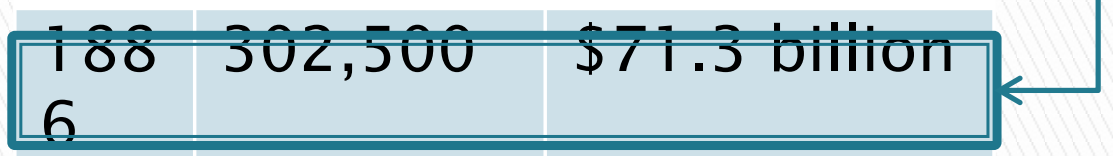

$190 \quad 22,500 \quad \$ 9.8$ billion 6

- "Invented for Life" Innovation

- 38,500 R\&D associates (2,500 in North America)

- Global R\&D expenditure: $\$ 5.8$ billion USD ${ }^{1}$

- A world leader in patent applications (4,126 in 2011)

- Ranked No. 1 in Germany, No. 5 in world 


\section{Research at Bosch}

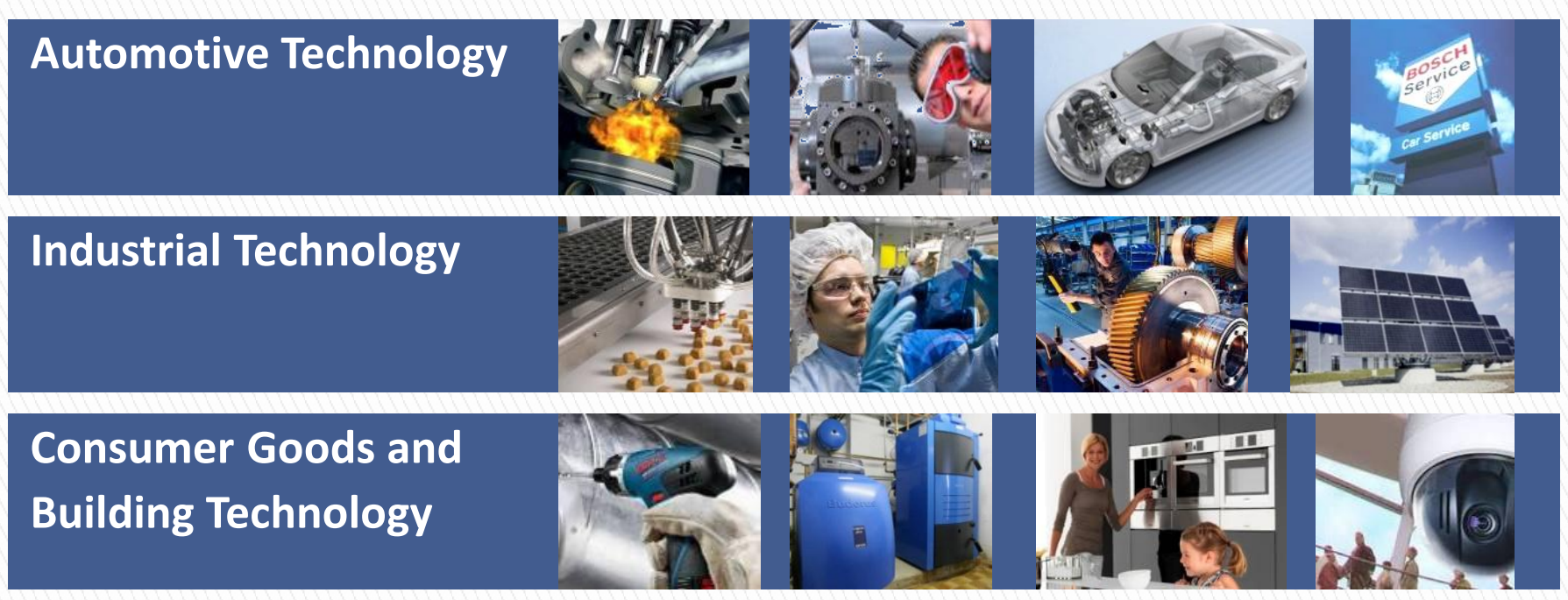

\section{- High Performance Computing at Bosch}

- Recent investment in terascale facilities ( 100 TFLOP)

- HPC development recognized as key for component development in energy sector

- Energy Conversion: Materials optimization; multi-scale, multiphysics coupled simulations

- Energy Storage: Materials discovery; Cell physics

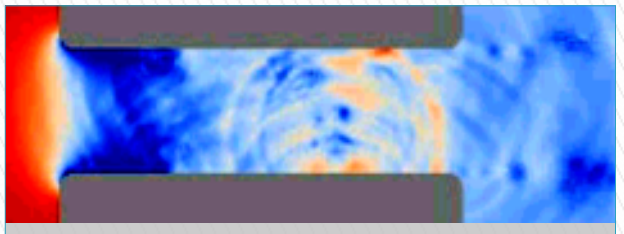

LES: Cavitation

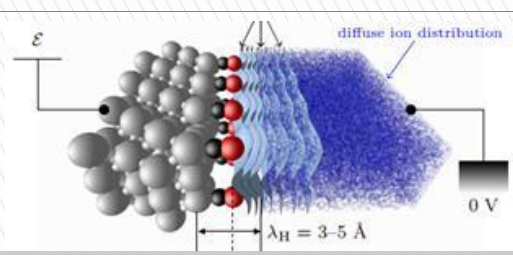

Ab-initio Simulations 


\section{Advanced Internal Combustion Engines}

- Advanced Combustion Concepts

- High efficiency (+30\%), low emissions

- Advanced control algorithms required for robust operation

- Multi-mode operation enables optimal operation over load map

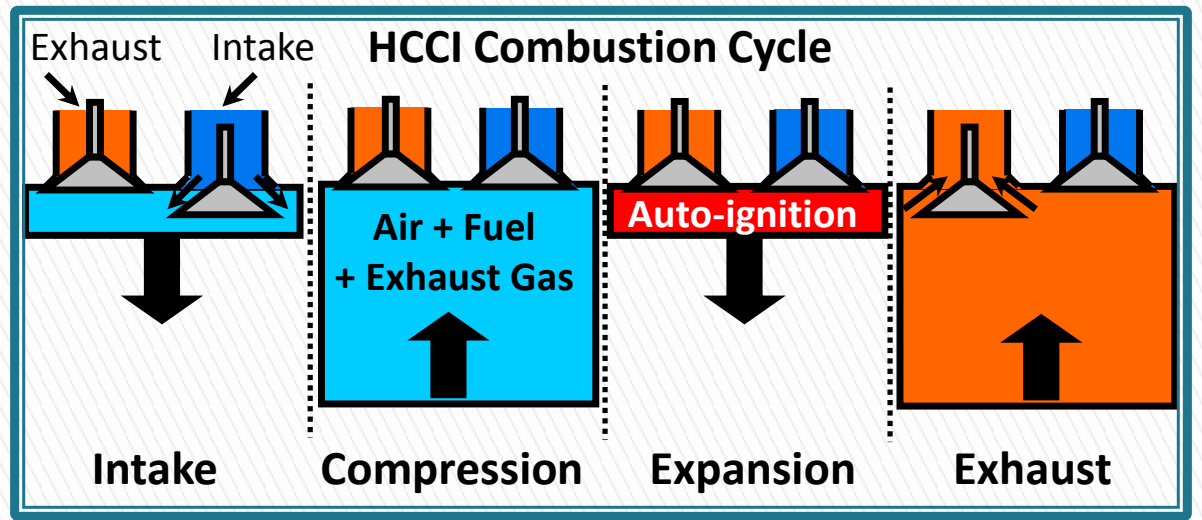

- Project Goals

- Understand the transient dynamics of switching between $\mathrm{SI}$ and $\mathrm{HCCl}$ combustion modes

- Aid the development of effective control strategies for multi-mode combustion engines

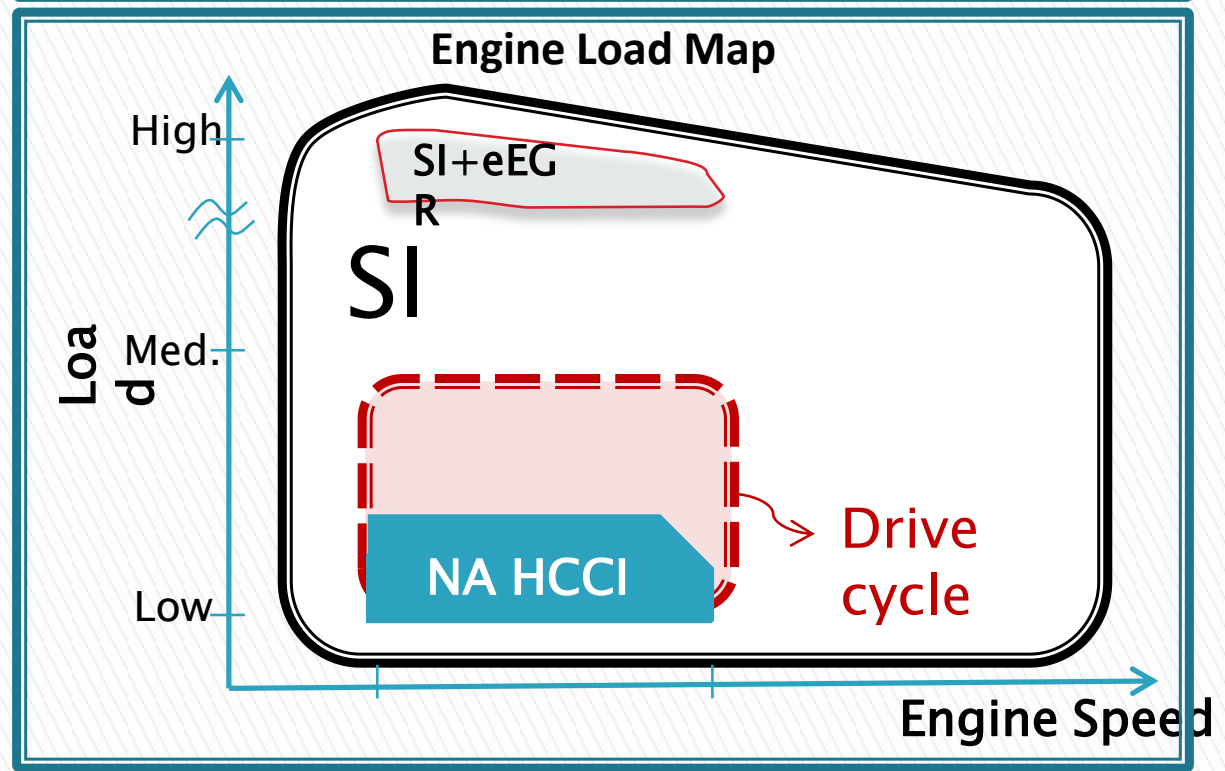




\section{Advanced Internal Combustion Engines}

- Modeling Approach

- Large Eddy Simulation (LES) of engine transition

- Structured explicit compressible code developed with Stanford U.

- Run on $1000-2000$ core

- 1.5-2 mio CPU-hrs/transition
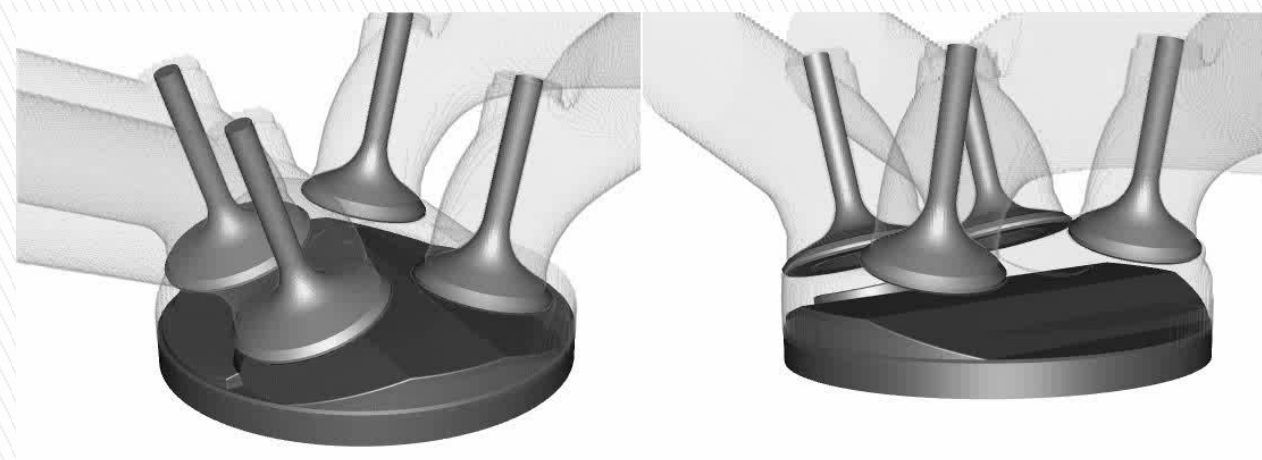

- Benefit of hpc4energy

- LES of transition feasible in timeframe relevant to make impact

- $70 \%$ reduction in time/cycle

- High fidelity critical to aid

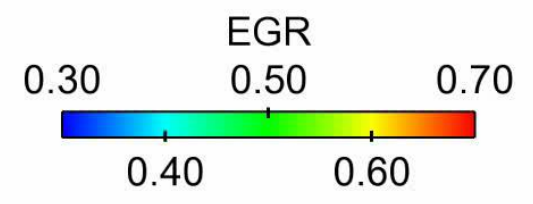
development of physics-based controller 


\section{Key Results}

- CAIO Combustion Code Run for SI to $\mathrm{HCCl}$ transition

- 4 days/engine cycle on 2000 cores

- Ran several different multi-cycle simulations, order $10^{7} \mathrm{CPU}$-hours utilized

- Evaluated CAIO code at higher resolution than previously possible - "Conditions under investigation" 
- Evaluated CAIO code at not previously obtainable levels of resolution

- Discovered limitations of CAIO combustion simulations at high resolution

- Learned that HPC is a valuable tool, but need codes better designed for large scale simulation 
- Gained insight into Bosch development process and needs

- Learned how to effectively and efficiently collaborate with industry in HPC

- Set framework for future collaboration with other partners (GE, Cummins, etc.)

- Continuing engagments in noncombustion activites 


\section{"Evaluation of Robust Unit Commitment" hpc4energy incubator wrap-up October 1st, 2013}

LLNL: Vera Bulaevskaya, Alan Lamont, Liang Min, Deepak Rajan, and Barry Rountree

ISO NE: Eugene Litvinov and Jinye Zhao

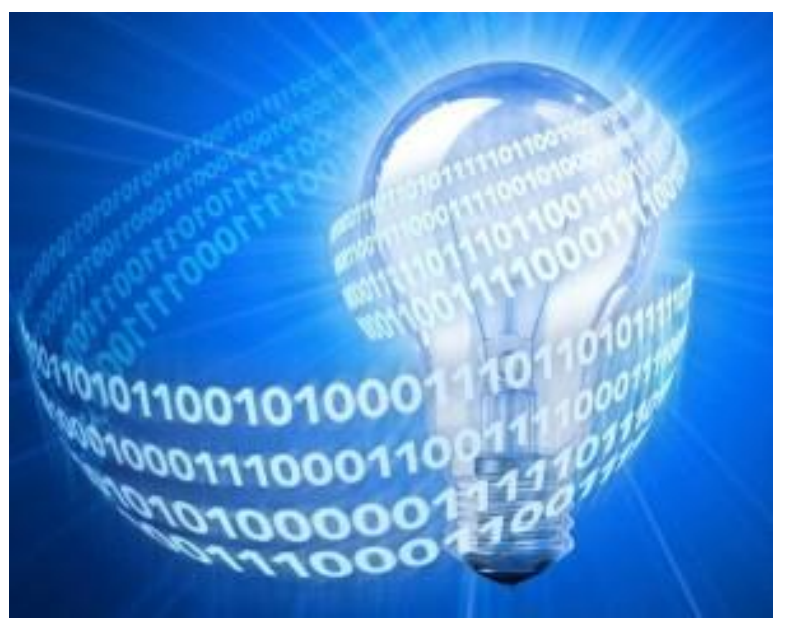

\section{"ISO New England"}

in collaboration with

Lawrence Livermore National Laboratory 


\section{Overview of ISO New Engliso newengand}

- 550 employees

- Three core responsibilities

- Ensure the day-to-day reliable operation of New England's bulk power generation and transmission system;

- Oversee and ensure the fair administration of the region's wholesale electricity markets; and

- Manage comprehensive, regional planning processes.

- Administer markets ranging from $\$ 5-\$ 11$ billion annually

- Operate 8,000 miles of HV transmission lines; 13 interconnections to electricity systems in New York and Canada

- Interests in High Performance Computing

- Internal production system using PSSE and TARA with Enfuzion in Transmission Planning and Operations Planning studies

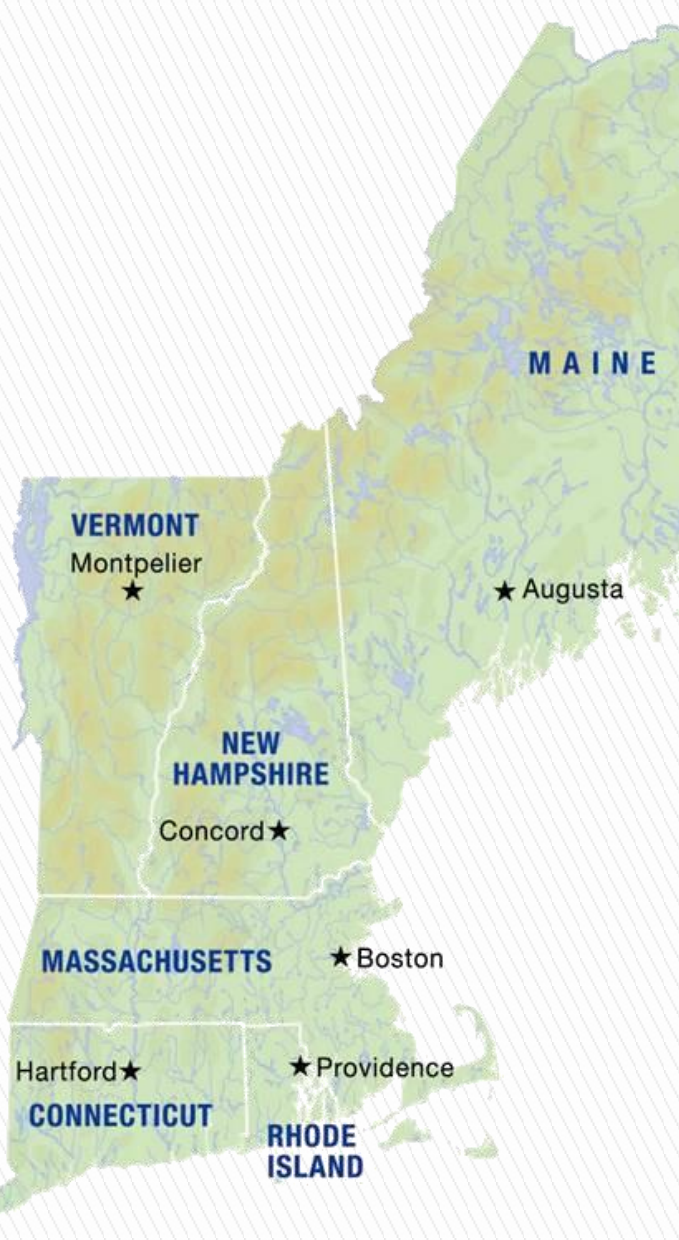

- Pilot project with Cycle Computing to use TARA and

Condor job scheduler at Amazon EC2

- R\&D project with EPRI on GPU based power flow apolication 


\section{Evaluation of Robust Unit Commitment}

- Benefits to the energy sector:

- Efficient computation enables ISO NE to perform more accurate and more comprehensive evaluation of the robust UC.

- Optimized generator operation and improved market efficiency.

- Project goal:

- Compare the robust approach with the deterministic approach by running a large number of Monte Carlo simulations.

- Evaluate the operational and economic benefits.

- Identify the optimal conservatism level of the robust approach. 


\section{Key Results}

Time to solve 7200 UC and

7.2 million ED problems

decreased from 600 days to

9.5 hours

- Generate random samples for simulations

- Number of historical data is small

- Use the "cluster method" to create clusters of samples

- Solve robust UC problems

- Multiple robust problems can be solved at the same time.

1000 ED runs for each robust UC

1 robust UC and 1000 ED runs per core

4800 cores used for 4800 robust UC and 4.8 million ED runs

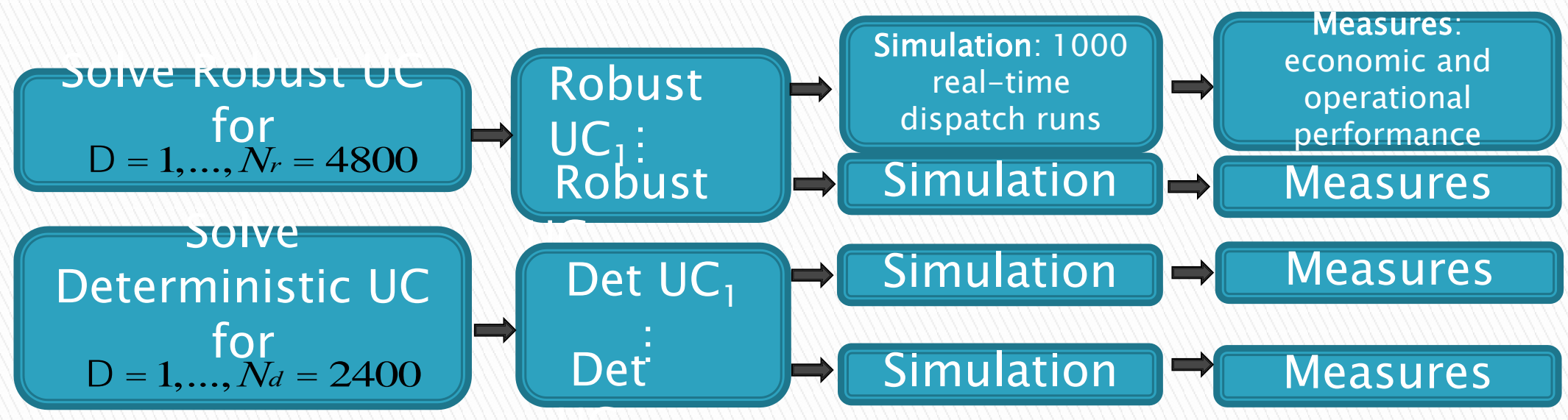


- HPC can efficiently run millions of simulations, which is impossible to be achieved by the computing capability of ISO

NE.

- Simulations yield more statistically significant results.

- Efficient computation enables ISO NE to perform more accurate and more comprehensive evaluation of the robust UC.

- LLNL provides expertise in HPC and statistics. 
- ISO-NE keeps promoting LLNL's value. This helps us build reputation in grid area.

- Engaging with ISO NE helps us understand better the R\&D needs at RTO/ISO community.

- ISO NE and LLNL are co-authoring an IEEE paper.

- We were approached by GAMS to scale up the simulation to 1 million ED runs for each UC. 


\section{Improving Models for Spray Breakup}

in Liquid Fuels Combustion hpc4energy incubator wrap-up October 2, 2013

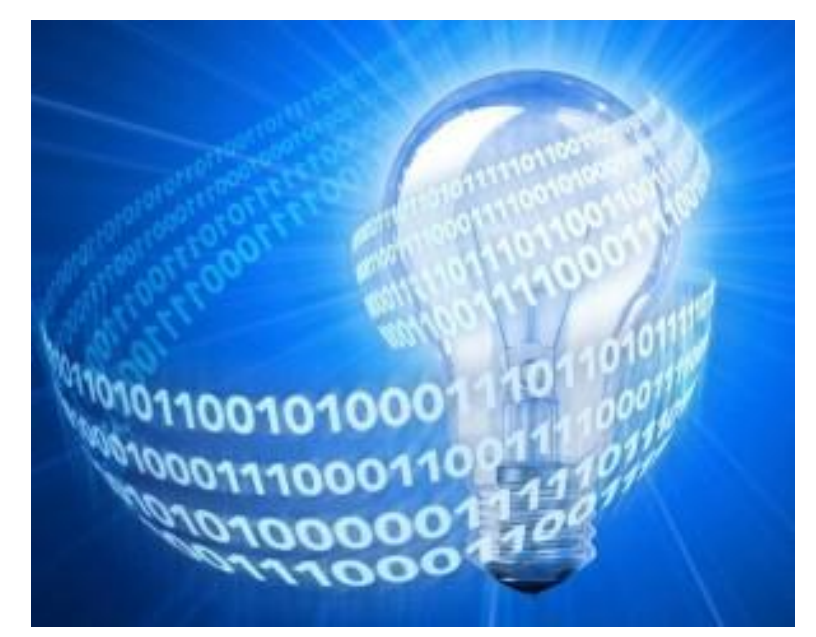

GE Global Research

in collaboration with

Lawrence Livermore National Laboratory

Cornell University

Arizona State University 
UL Ulunal nesedill. splay Breakup

GE jet engine design issues

- Efficiency, reliability, durability, minimizing pollutant formation

- Little current use of HPC

- Still designing with older paradigms \& methods

Project goal Jet Turbine \& Combustor

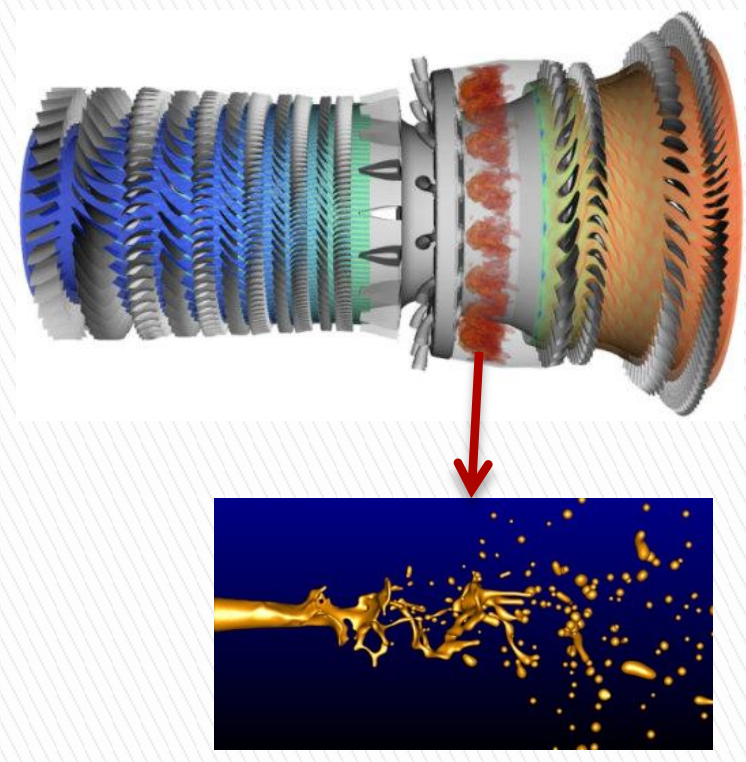

Liquid Fuel Breakup

- HPC to study realistic fuel injector designs

- Evaluate computational approaches to model complex geometries

- Identify HPC needs for predictive spray breakup simulations 


\section{Key Results: Liquid Jet in Cross Flow}

Prior Simulations: complex geometry but low fidelity models

HPC4Energy simulations
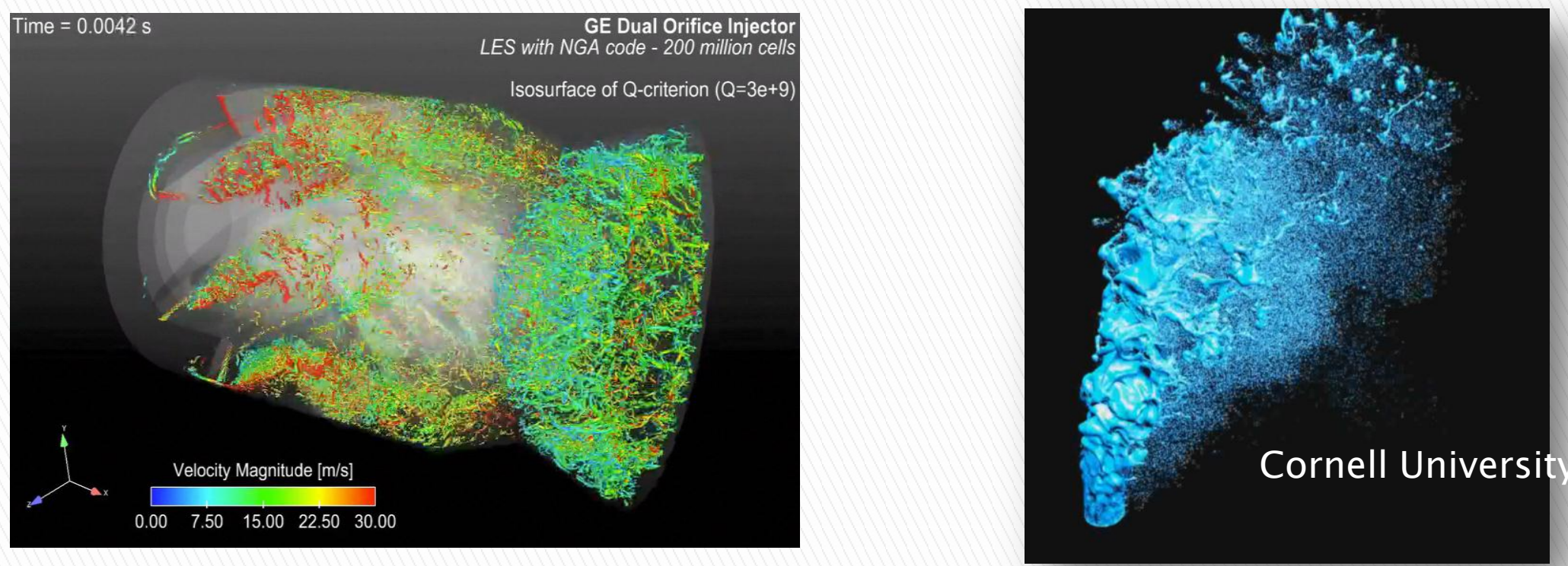

- Simulations capture physics consistently with experiment

- Richer physics with better models and denser mesh 


\section{Recearrh}

- Simulation sizes not normally run in an industrial setting

- Insight into better models for JIC in near-injector and dense spray regions

- Potentially fewer, better designed experiments to shorten design and development process

- Provides evidence for value of HPC within company

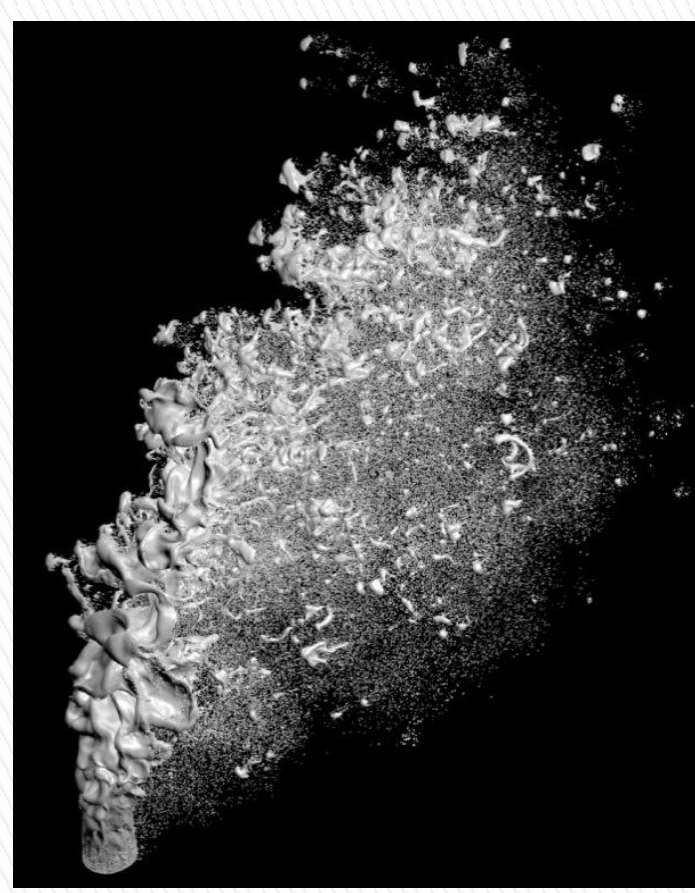


- Perspective of industry applications involving spray breakup, and what is the current stateof-the-art.

- Access to source algorithm allows us to "pull apart" and learn about these state-of-the-art numerical techniques, and continuing collaboration with academia

- Codes are ported to HPC machines, configured for $+10 \mathrm{~K}$ core runs, now available for LLNL work through TASC (tasc.IInl.gov)

- Future: Other multiphase flows at LLNL, e.g., Advanced Rocket-Engine Design (DARPA) 


\section{BACK UP SLIDES}


Validation of JIC numerical simulations with

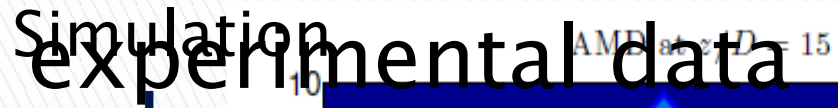

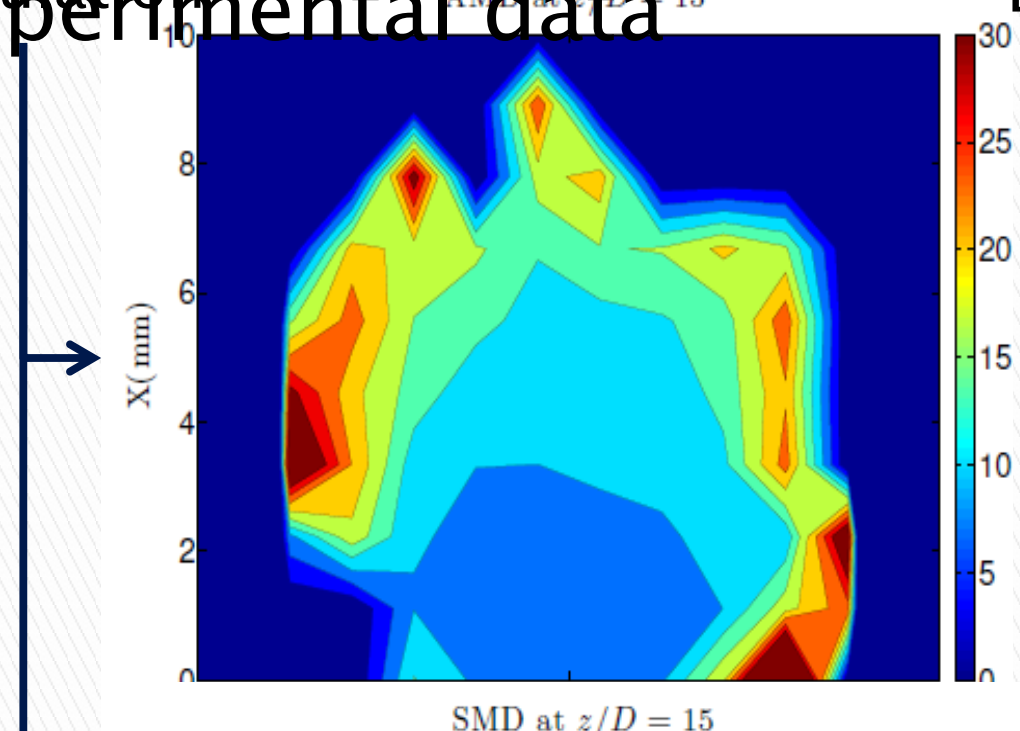

Experiment
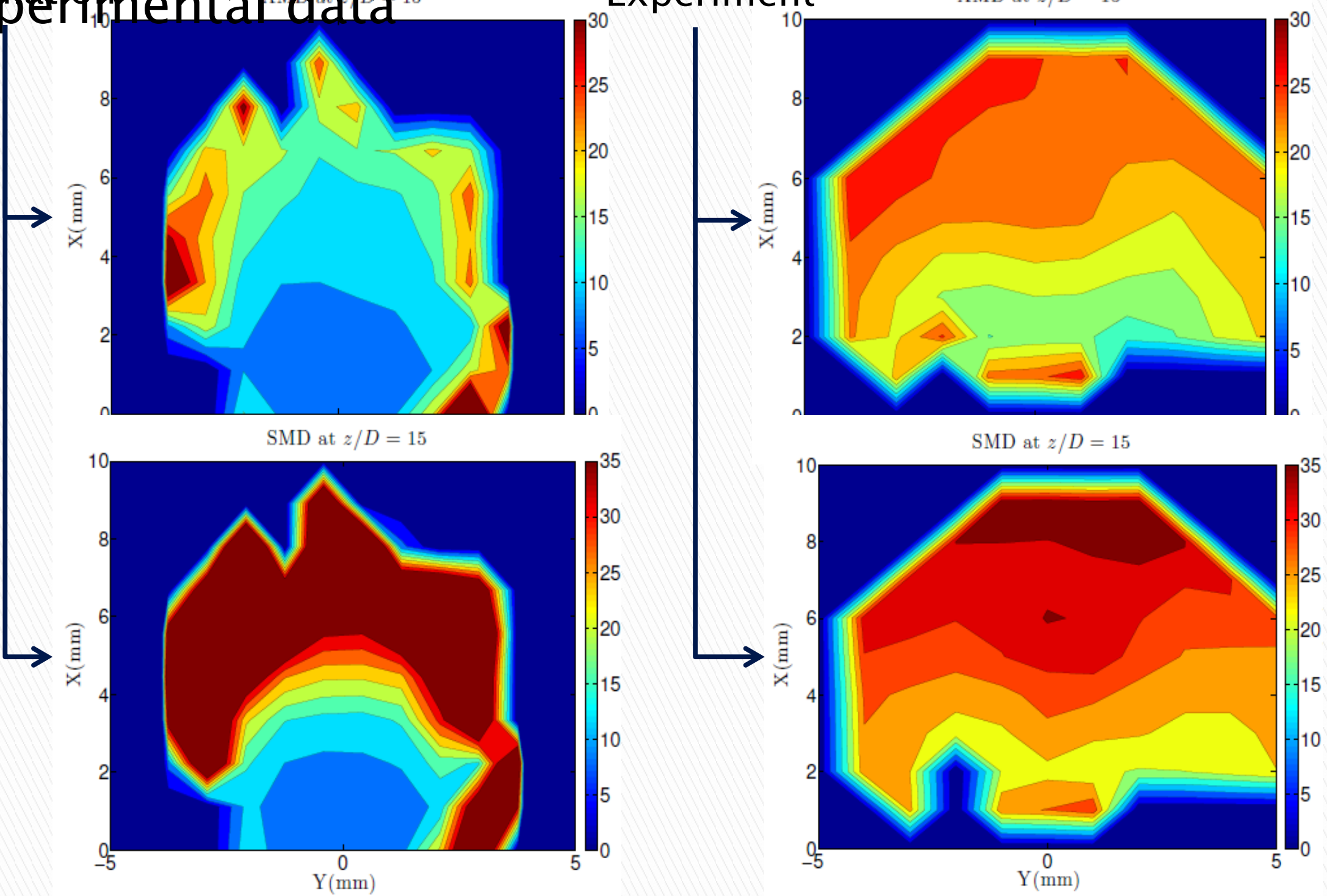


\section{Improving Thermal Spallation Drilling}

\section{with HPC Simulations hpc4energy incubator wrapup October 2, 2013}

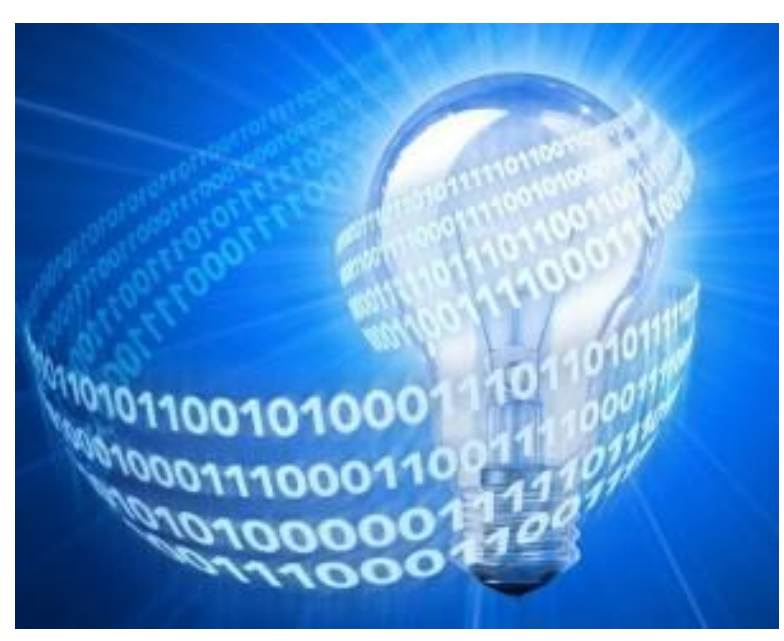

Potter Drilling

in collaboration with

Lawrence Livermore National Laboratory 


\section{Potter Drilling}

- Cleantech start-up

- Developing innovative drilling and rock excavation technologies for revolutionary performance in hard rocks

- Geothermal, oil \& gas, mining

- Only have access to workstation-scale CFD and FEA

- Need HPC to complement and reduce empirical development

- High cost and long time associated with field trials

- Challenge of observing/measuring downhole process

- Determining changes in process parameters for differing rock types

- Extrapolating to greater depths and conditions not feasible for testing 


\section{Thermal Spallation: An Effective Way to Excavate Hard Rock

1. Intense heat creates high stress at the rock surface

2. Micro-fractures initiate at flaws or mineral boundaries

3. To relieve stress, spall is ejected, exposing fresh surface
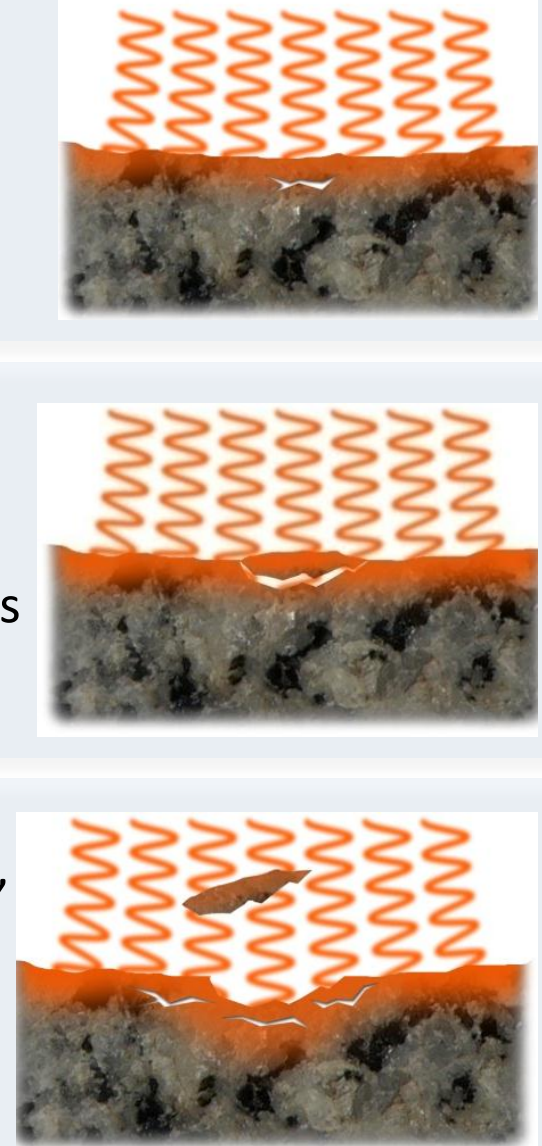
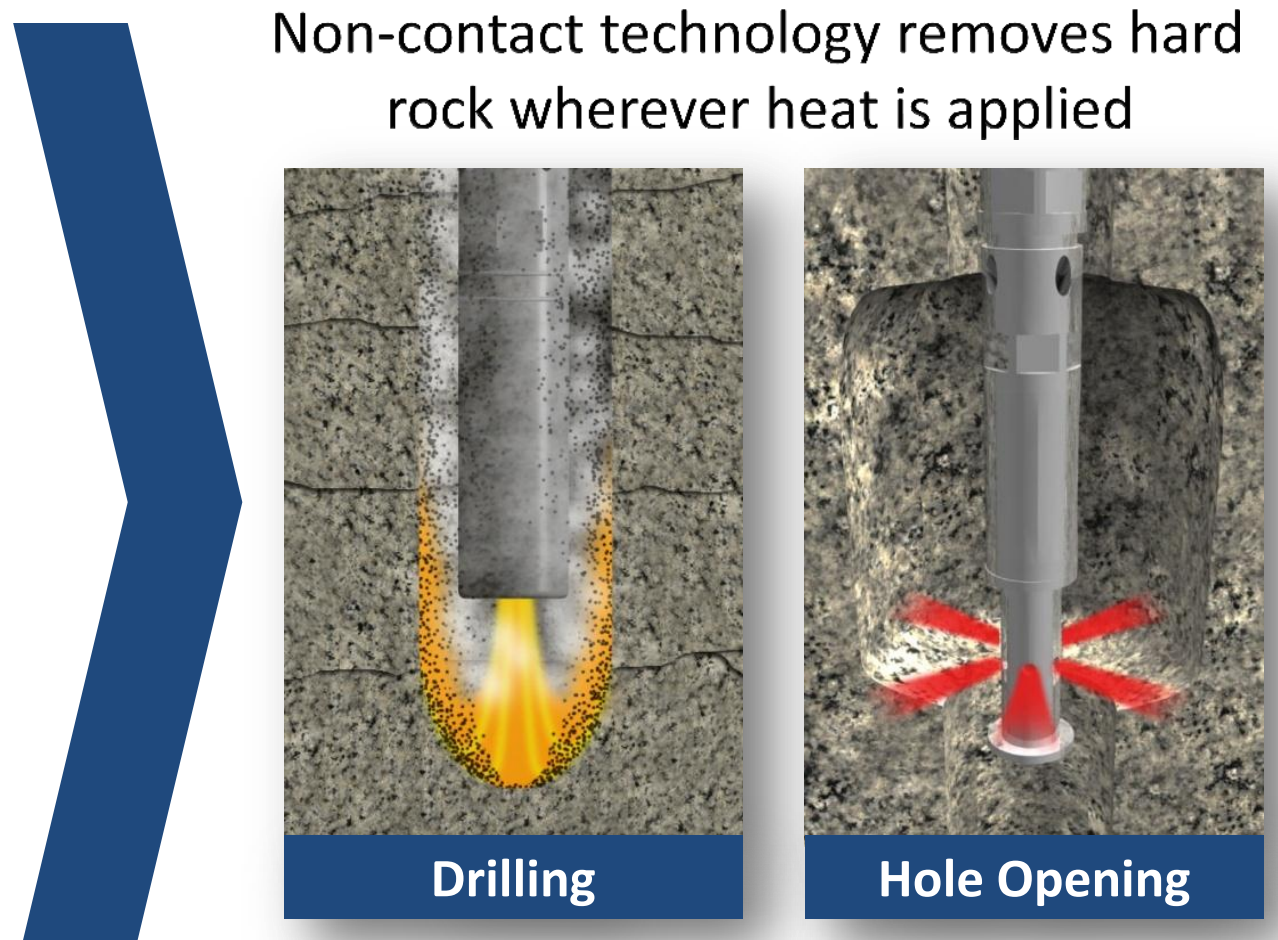


\section{Project Description}

- $\quad$ HPC for Thermal Spallation Drilling

- Potential to decrease cost of drilling hydrothermal wells by up to $25 \%$ (value of $\$ 1 \mathrm{M} /$ well)

- Net increases in geothermal productivity by at least $2 x$ (value of $\$ 5$ 7M/well)

- Enables more widespread Engineered Geothermal Systems (EGS)

- Applications to oil \& gas: decreases hard rock drilling costs, increased well productivity, EOR, non-hydraulic fracking, and more.

- Scope of Work

- Conduct a detailed parametric analysis of the factors impacting thermal spallation of hard basement rocks.

- $\quad$ Extract the necessary parameters (spall sizes, critical flaw distributions and critical stress thresholds etc.) needed to validate parametric models 


\section{Results}

- Finished Stage $1 \& 2$ simulations

- 2D: Completed over 7000 simulations 200K CPU-hrs; 72 processors per simulation

- 3D: Completed 50 simulations 500K CPU-hrs; 1020 processors per simulation

- Five sets of 2D parametric runs have been completed and analysed

- Examined: borehole conditions, microstructural properties, thermal and mechanical properties.

- Determined factors most crucial to damage extent and spall size.

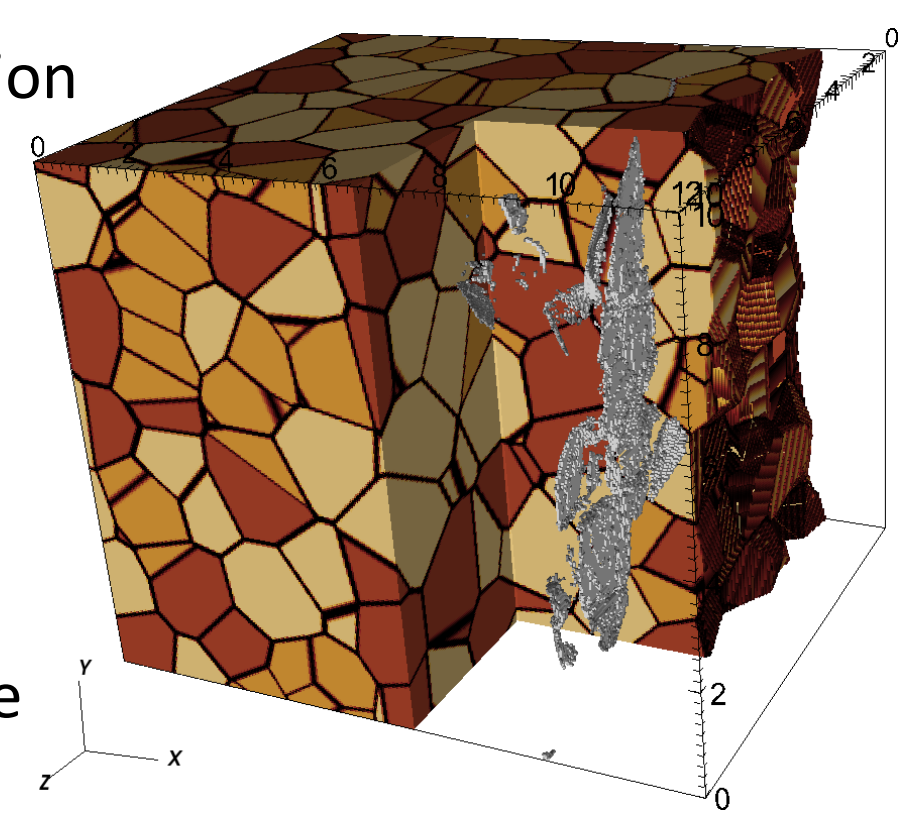

- Results used to constrain 3D parameter space and reduce number of runs. 


\section{Value Added by hpc4energy Collaboration}

- Providing valuable insights into the actual mechanism for spall formation

- Identifying most critical parameters for optimizing process

- Will allow for extrapolation to depths and conditions not feasible by empirical approach

- HPC resources not otherwise available to small or start-up companies

2000 simulations (1 per point)

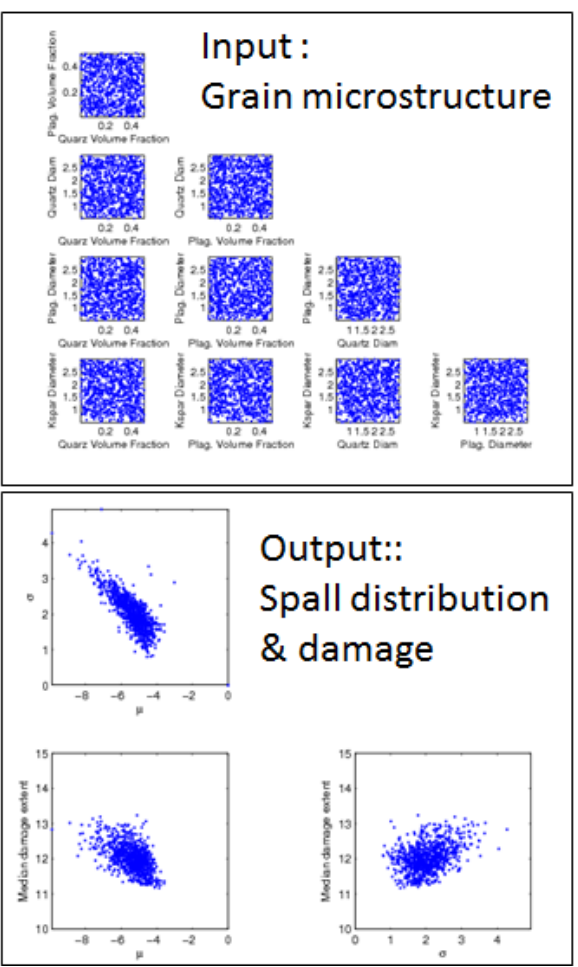




\section{Value Added by Collaboration to LLNL}

- Better understanding of effective strategies and challenges when working with small businesses

- Promotion of LLNL's HPC capabilities to the geothermal and drilling communities

- Implementation and validation of a new GEODYN-PSUADE interface to control simulation runs and analysis. 


\section{"Improving PSLF Simulation Performance and Capability"}

hpc4energy incubator wrapup

October 2, 2013

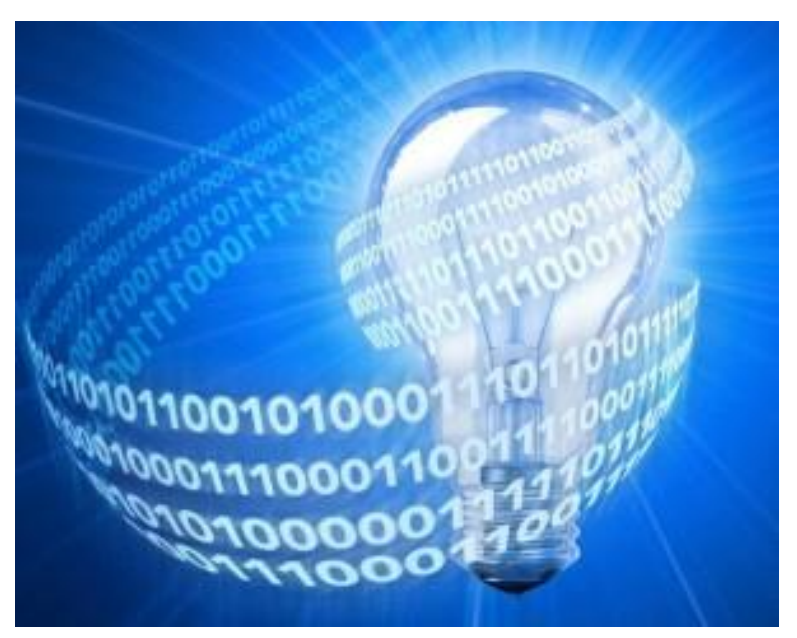




\section{GE Energy Overview}

- $\sim$ \$4 billion/yr

- 100,000 employees

- Software $\sim 3 B$

- Main business segments

- Energy Management

- Oil and Gas

- Power and Water
- GE Concorda suite of tools

- Planning and simulating electric power grids

- Assessing economic performance

- Evaluating generation reliability.

- Deployed to 130+ companies

- $\quad$ PSLF transmission planning component

- Steady state and dynamic analysis

- Study impacts of system events

- The hpc4energy project focused on contingency analysis performance

- Scaling

- Computational efficiency

- Current runtimes are hours to days

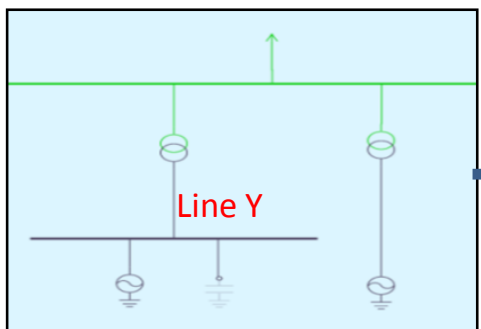

Base Case
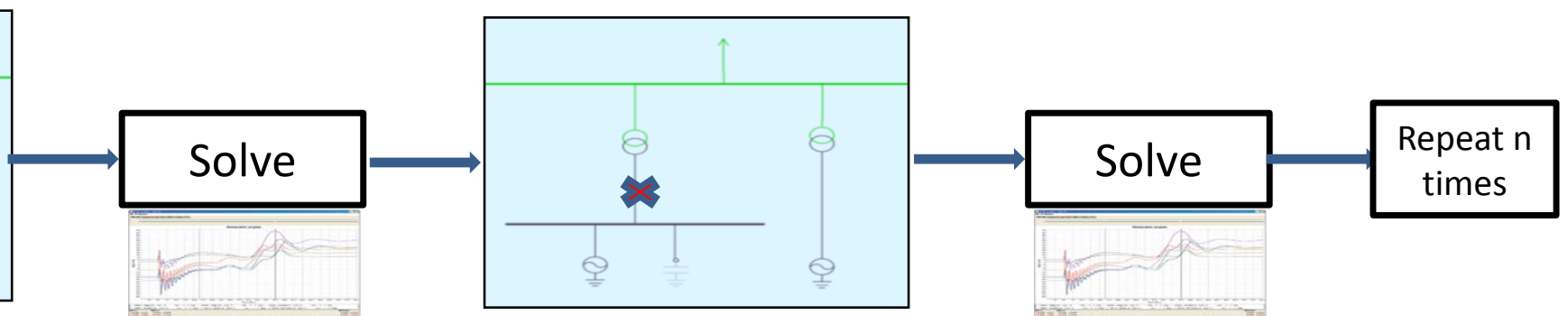

Contingency - Outage of Line $Y$

Post-processing 


\section{Benefits to Industry}

- Improve ability to identify potential major system reliability concerns

- Improve evaluation of emerging clean energy technologies

- More accurate models with greater than 150,000 buses

- Focus on results analysis and less time on performing analysis has a direct financial benefit for utilities 


\section{Key Results}

- Parallel execution of contingencies enables larger more complete studies

- Large number of contingencies $(4,217)$; current norm is 100 's

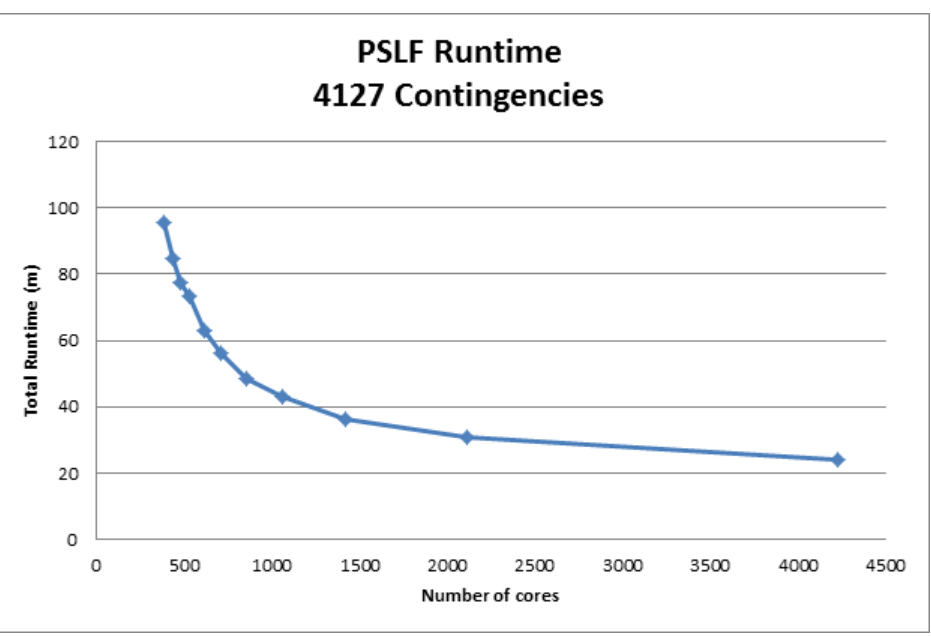

Reduced runtime from 23.5 days to 23 minutes

- Significantly improved matrix reordering algorithm solution times through optimization and threading

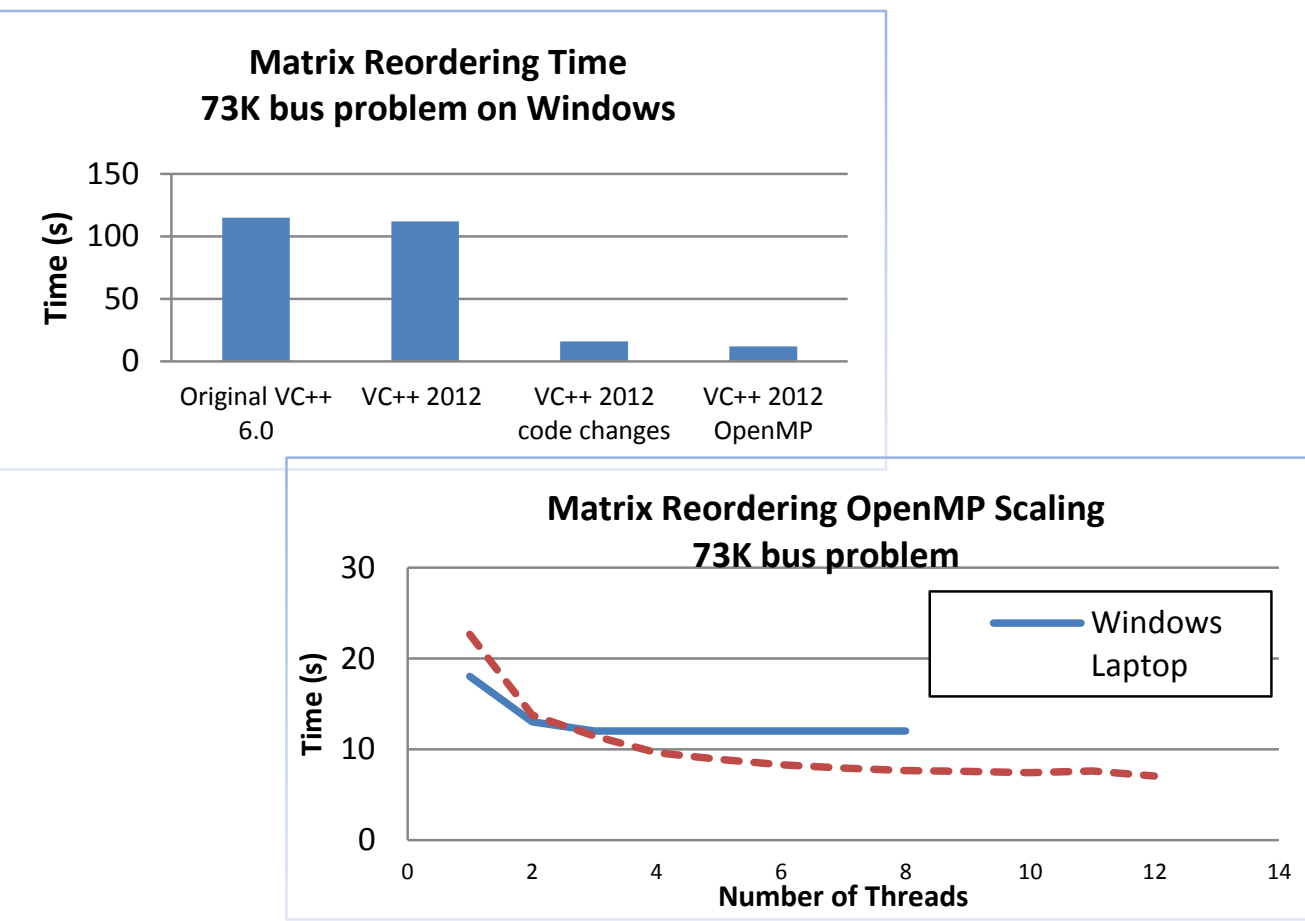

Reordering speedup of 9.6 times on Windows and 7.2 times on Linux 


\section{Value added by collaboration to LLNL}

- Built relationships and credibility

- Demonstrated how LLNL HPC expertise can be applied to an industry application

- New domain for applying LLNL developed solvers

- Technical experience and knowledge

- Application used by CA utilities

- Looking into a power system modeling application

- Porting Windows applications to HPC

- Running thousands of instances of an application 


\section{"Building System Models to Enable Deep}

\section{Energy Retrofits"}

hoc4energy incubator wrap up

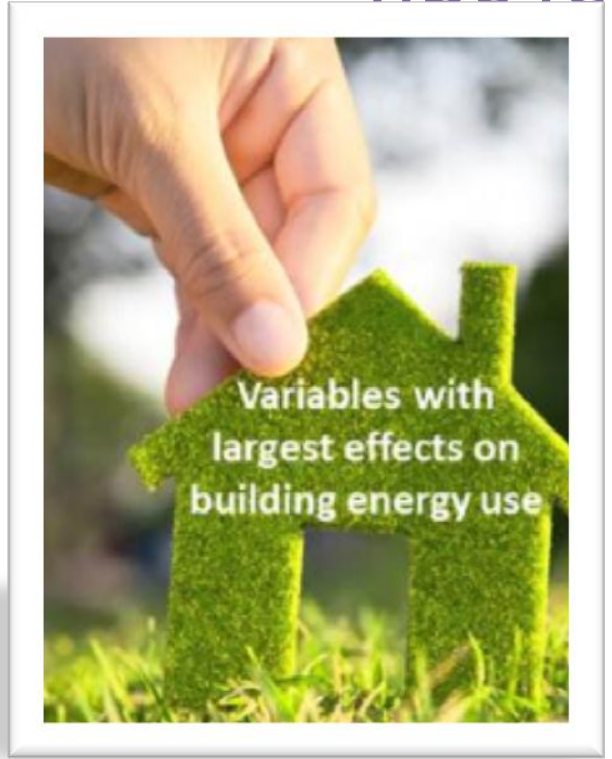

October1, 2013

in collaboration with

Lawrence Livermore National Laboratory

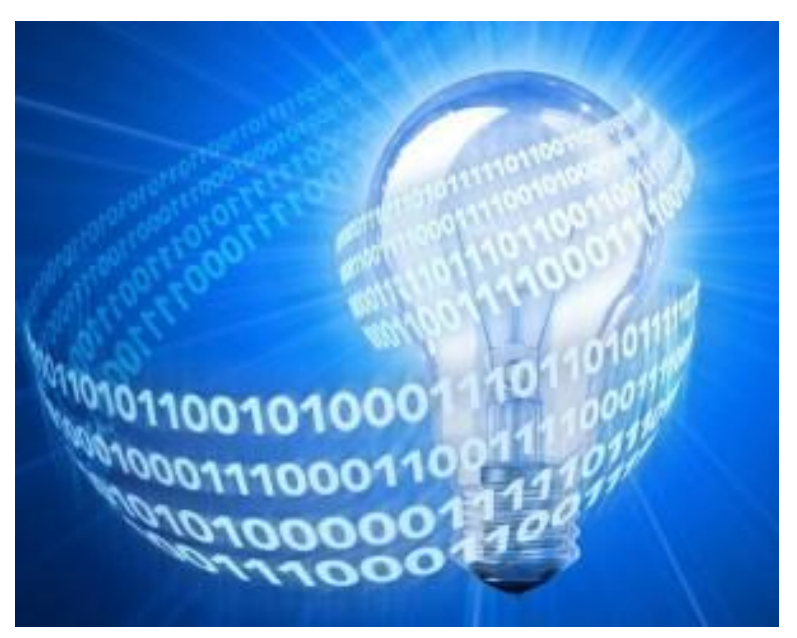




\section{UTRC Research}

- $\$ 60 \mathrm{~B}$ in revenue (UTC), 1,000 employees (UTRC)

- UTC: Carrier, UTC Aerospace, Otis, Pratt \& Whitney, Sikorsky Aircraft, UTC Fire \& Security

- UTRC: Research arm of UTC

- UTRC: An unclear HPC investment strategy

- Currently use computing at giga-scale

- UTRC, Sikorsky, others....

- Buildings use $40 \%$ of energy in the US annually

- Buildings typically use up to $30 \%$ than designed 


\section{Key Results}

- Building Energy Simulation

- Identify most impactful parameters

- Speed up process

- ROM, UQ via PSUADE

- UTRC ran over 10,000 runs on Sierra

- Each run used 1,000 cores

- Processing time reduced by a factor of 60

- Only 50 of 917 parameters impacted the simulation

- Model calibration: error reduced to 5\%

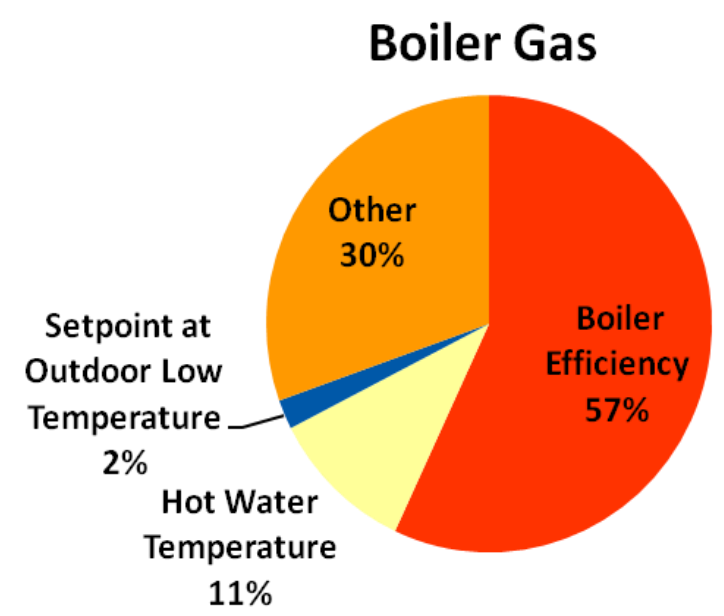




\section{Value added by HPC/LLNL}

collaboration to the Companv

- Project improvement:

- Reduced time

- Expanded parameter set

- HPC is a great resource for UTC

- LLNL collaboration can help improve their computational, analytical, and algorithmic capabilities 


\section{Value added by collaboration to LLNL}

- Better understanding of UTC

- Established expertise in the Building Simulation domain

- A real champion in HPC and LLNL from UTC

- Expansion of LLNL in EEB Hub, leading to EERE projects 